
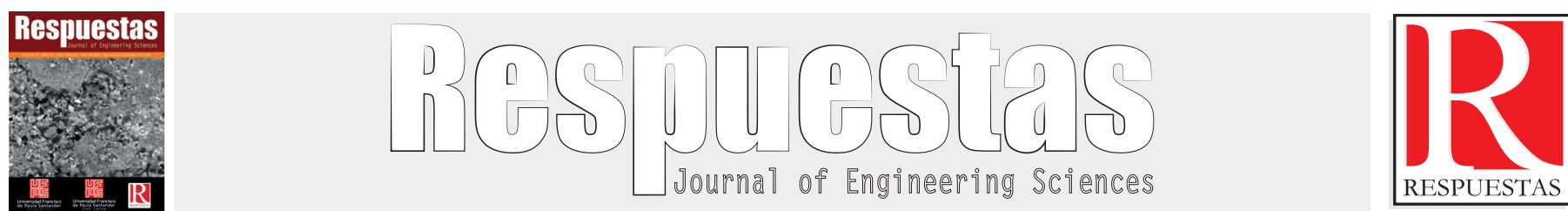

Original Article

https://doi.org/10.22463/0122820X.1897

\title{
Elaboración Y Caracterización De Bloques Cerámicos Extruídos Usando Cenizas De La Combustión De Carbón A Escala De Laboratorio
}

\author{
Elaboration And Characterization At Laboratory Scale Of Extruded Ceramic Blocks Using Carbon \\ Combustion Ashes \\ Karen Bibiana Díez Contreras ${ }^{1}$, Jorge Sánchez Molina², Diana Alexandra Torres Sánchez ${ }^{3}$ \\ ${ }^{1}$ Maestría en Química, karen.diez@unipamplona.edu.co, Universidad de Pamplona, Pamplona, Norte de Santander, Colombia. https://orcid.org/0000-0002-8735-9600 \\ ${ }_{2}^{2}$ PhD en Avances en Ingeniería de los Materiales y Energías Sostenibles, jorgesm@ufps.edu.co, orcid.org/0000-0002-9080-8526, Universidad Francisco de Paula Santander, Cúcuta, \\ Norte de Santander, Colombia. \\ ${ }^{3}$ Doctora en Ciencias - Química, datorres@unipamplona.edu.co, orcid.org/0000-0002-1733-2904, Universidad de Pamplona, Pamplona, Norte de Santander, Colombia.
}

How to cite: K.B Diez-Contreras, J. Sanchez-Molina, D.A. Torres-Sanchez, "Elaboración Y Caracterización De Bloques Cerámicos Extruídos Usando Cenizas De La Combustión De Carbón A Escala De Laboratorio”. Respuestas, vol. 25, no. S1, pp. 28-35, 2020.

Received on August 30, 2019; Approved on November 7, 2019

\begin{tabular}{ll}
\hline & ABSTRACT \\
\hline Keywords: & $\begin{array}{l}\text { Ashes resulting from the combustion of coal used to feed the hive furnaces of the ceramic industry of Norte } \\
\text { de Santander, contain potentially hazardous pollutants for the environment and have no practical application } \\
\text { so far, for that reason they become a waste material. In this study, a formulation was developed to make } \\
\text { a ceramic material composed of a mixture of clay from Norte de Santander (Colombia) and ash residues } \\
\text { from the combustion of coal used to feed hive kilns in the region, which were added in proportions of } 10,\end{array}$ \\
Clay, & 20 and $30 \% \mathrm{w} / \mathrm{w}$, forming prototype mixtures of extruded and sintered ceramic blocks in a temperature \\
coal ash, & $\begin{array}{l}\text { range between } 900 \text { to } 1100^{\circ} \mathrm{C}, \text { with gradients of } 50^{\circ} \mathrm{C} \text {. FTIR-ATR was used to identify the presence of } \\
\text { different functional groups and the mineralogical composition was studied by DRX of the raw materials; }\end{array}$ \\
ceramic blocks, & $\begin{array}{l}\text { Likewise, the technological behavior of the developed mixtures was evaluated. The results allowed to select } \\
\text { the mixture in which } 10 \% \text { of coal ashes and a cooking temperature of } 9500^{\circ} \mathrm{C} \text { were added, since it has }\end{array}$ \\
DRX,ATR, & a percentage of water absorption and a compressive strength according to the ranges established by the \\
technological behavior. & Colombian standards, generating as an added value the lightening and reduction of the cooking temperature, \\
becoming an alternative of innovation and development that can be used in the production processes of \\
traditional materials of the region with an ecological seal.
\end{tabular}

\section{RESUMEN}

Palabras clave:

Arcilla, cenizas de carbón, bloques cerámicos, FTIR-ATR, DRX, comportamiento tecnológico.
Las cenizas producto de la combustión del carbón utilizado para alimentar los hornos colmena de la industria cerámica de Norte de Santander, contienen contaminantes potencialmente peligrosos para el medio ambiente y hasta el momento no tienen ninguna aplicación práctica, por lo cual, se convierten en un material de desecho en el departamento. En este estudio, se desarrolló una formulación para realizar un material cerámico compuesto de una mezcla de arcilla de Norte de Santander (Colombia) y residuos de cenizas provenientes de la combustión del carbón utilizado para alimentar hornos colmena de la región, las cuales fueron adicionadas en proporciones de 10, 20 y $30 \%$ en peso, conformando con las mezclas prototipos de bloques cerámicos extruídos y sinterizados en un rango de temperatura entre 900 a $1100^{\circ} \mathrm{C}$, con gradientes de $50^{\circ} \mathrm{C}$. Se usó FTIRATR para identificar la presencia de diferentes grupos funcionales y se estudió la composición mineralógica mediante DRX de las materias primas; así mismo, se evaluó el comportamiento tecnológico de las mezclas desarrolladas. Los resultados permitieron seleccionar la mezcla en la que se agregó el $10 \%$ de cenizas de carbón y una temperatura de cocción de $950{ }^{\circ} \mathrm{C}$, ya que presentó un porcentaje de absorción de agua y una resistencia a la compresión acorde a los rangos establecidos por las normas colombianas, generando como valor agregado el aligeramiento y reducción de la temperatura de cocción, convirtiéndose en una alternativa de innovación y desarrollo que se puede utilizar en los procesos de producción de materiales tradicionales de la región con un sello ecológico 


\section{Introducción.}

Los bloques cerámicos desde hace décadas se han producido principalmente a partir de arcilla pura; sin embargo, la extracción continua de arcilla y la remoción de la capa superior del suelo para la fabricación de bloques causa un agotamiento sustancial de este recurso natural (Castells, 2015) (Çiçek \& Çinçin, 2015).

En el área metropolitana de Cúcuta, la mayoría de los productos cerámicos se fabrican en hornos colmena o de llama invertida, siendo su utilización un $77 \%$ que corresponde a aproximadamente 220 hornos de este tipo, los cuales se pueden encontrar en diferentes tamaños sin presentar variaciones significativas en sus características de diseño (Sánchez Molina \& Ramírez Delgado, 2013). En las empresas de cerámica de la región, el material utilizado como combustible para la operación de estos hornos es el carbón dosificado principalmente por operarios y de donde se producen cenizas como residuos del carbón quemado durante la combustión en las hornillas (Sánchez \& Díaz, 2011). Las cenizas producidas por la combustión de carbón en los hornos cerámicos industriales de Norte de Santander (Colombia) contienen sustancias contaminantes que son potencialmente peligrosas para el medio ambiente y debido a que no tienen aplicaciones prácticas, se convierten en materiales de desecho (Lafarge, 2014).

La inclusión de cuerpos no arcillosos desde un punto de vista cerámico tiene ciertos límites, y se deben evaluar las cantidades máximas de estos materiales ajenos (nutrientes) que pueden ser incorporados a la mezcla de arcilla para elaborar cerámica estructural, sin cambios en la calidad de los productos (Castells, 2015). Si el nutriente tecnológico entra a formar parte químicamente del nuevo compuesto su futuro comportamiento será bueno; pero si el nutriente solo es retenido físicamente sin cambio alguno en su estructura morfológica y/o química, quedará encapsulado y su futura evolución dependerá más del medio donde se halle que de su propia estabilidad y resistencia. En el primer caso se tratará de una ceramización, que viene a ser la verdadera inertización, mientras que en el segundo escenario se deberá hablar de encapsulación (Arsenovic, Radojević, Jakšić, \& Pezo, 2015) (Consoli, Rocha, \& Saldanha, 2014).

El uso de nutrientes tecnológicos en la industria cerámica ha sido un tema de investigación a nivel internacional, de- bido a que ambientalmente existe la necesidad de generar una disposición de los residuos, que sean recirculados y/o usados como aditivos para la fabricación de nuevos productos y con esto disminuir el consumo de materias primas naturales. Entre los nutrientes tecnológicos que han sido estudiados para la fabricación de nuevos materiales cerámicos, se encuentran las cenizas de incinerador de biomasa, residuos maderables, tierra blanqueada de la industria del petróleo, lodos de aguas residuales urbanas, bagazo de caña de azúcar, lodos de la industria cervecera, aguas residuales del molino de aceituna, residuo de café molido, cenizas de cisco de café, cascarilla de arroz, ceniza de la cascarilla de arroz, residuos de la industria del papel, residuos de producción de biodiesel, lodo rojo derivado de la industria del aluminio, cenizas volantes de la combustión del carbón, entre otros (Castells, 2015) (Sánchez Molina, Corpas, \& Álvarez Rozo, 2019) (Vidal, Torres, Mejia, \& Gonzalez, 2012) (Barrera Castro, 2009) (Rozo, 2013) (Balaguera \& Carvajal, 2004) (Monroy \& Mora, 2005) (Durán Angarita \& Pinto Gelvez, 2001) (Díaz Fuentes, 2014).

Para el campo cerámico en específico, el uso de cenizas de carbón que se ha reportado corresponde a las cenizas volantes provenientes de proceso de combustión del carbón de las termoeléctricas, obteniéndose cuerpos cerámicos con propiedades como la contracción, la absorción de agua y la resistencia similares y en algunas ocasiones mejores a la de los materiales cerámicos comerciales que se utilizan para baldosas y pavimentos; sin embargo, se evidencia la aparición de corazón negro en las muestras cocidas rápidamente a altas temperaturas. (Kockal, 2012) (Díaz Fuentes, 2014) (Yang, Shili, Shuhua, Chunli, \& Xiaohui, 2017) (Sarabia, Ramirez, \& Sánchez, 2018).

Teniendo en cuenta que las cenizas de la combustión del carbón de hornos colmena y los inquemados de carbón generados en el proceso, han sido objeto de un estudio reciente (Sánchez Molina et al., 2019), la presente investigación es pionera en la inclusión de estos para la fabricación de bloques cerámicos extruídos, los cuales presentaron mejores propiedades tecnológicas a temperaturas menores que las usadas actualmente para la cocción de los mismos.

\section{Materiales y métodos.}

A nivel industrial, las empresas del sector cerámico adi 
cionan el carbón utilizado para las quemas por medio de operarios (dosificación con pala) y por medio de carbojet (dosificación mecánica); en este estudio, se recolectaron cenizas mezcladas de una empresa de la región (figura 1) provenientes de la combustión de carbón de los hornos

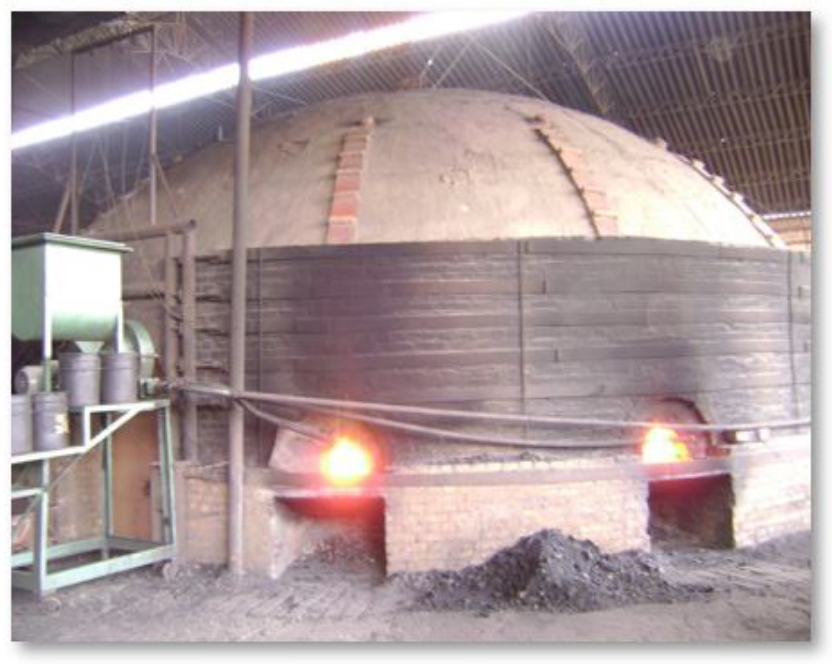

colmena, se molieron en un molino de martillos y se tamizaron en malla ASTM 40. La arcilla fue recolectada en la mina La Alejandra ubicada en el municipio de El Zulia en Norte de Santander, Colombia.

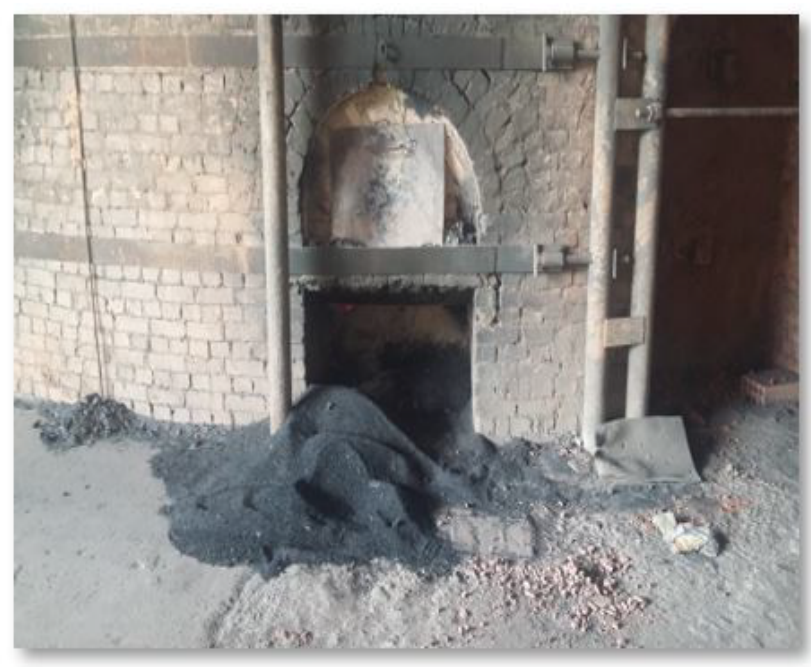

Figura 1. Recolección de cenizas de carbon de los hornos colmena Fuente: Autores

La arcilla se mezcló con la ceniza en proporciones de 10 , 20 y $30 \% \mathrm{p} / \mathrm{p}$, para construir prototipos de bloques que se extruyeron en una extrusora de vacío New Wave serie 101 y se cocieron en un rango de temperatura entre $900 \mathrm{a}$ $1100{ }^{\circ} \mathrm{C}$, con gradientes de $50{ }^{\circ} \mathrm{C}$ Se usó FTIR-ATR (IR Prestige-21, Shimadzu, Japón) para identificar la presencia de diferentes grupos funcionales y se estudió la composición mineralógica mediante DRX (difractómetro de polvo, Bruker, D8 ADVANCE con Geometría DaVinci) de las materias primas; así mismo, se evaluó el comportamiento tecnológico de las mezclas desarrolladas según la norma NTC 4017 (ICONTEC, n.d.-a).

\section{Resultados y discusión.}

\section{Análisis mineralógico.}

Con el propósito de determinar los grupos funcionales principales de las materias primas empleadas en la elaboración de los prototipos de bloques cerámicos, se utilizó la espectroscopía infrarroja con transformada de Fourier (FTIR), que además sirve como complemento de los análisis mineralógicos realizados por DRX, ya que FTIR es una técnica que permite la identificación de los cambios tanto de las fases cristalinas como de las fases amorfas, en tanto que DRX se limita a las fases cristalinas (Madejová, 2003) (Shoval \& Beck, 2005).

\section{Difracción de rayos $\mathrm{X}$ de la muestra de arcilla.}

La caracterización mineralógica de la muestra de arcilla se realizó mediante difracción de rayos X (DRX). En la figura 2 se muestra el patrón de difracción obtenido y en la tabla 1 el análisis mineralógico cuantitativo realizado mediante refinamiento Rietveld.

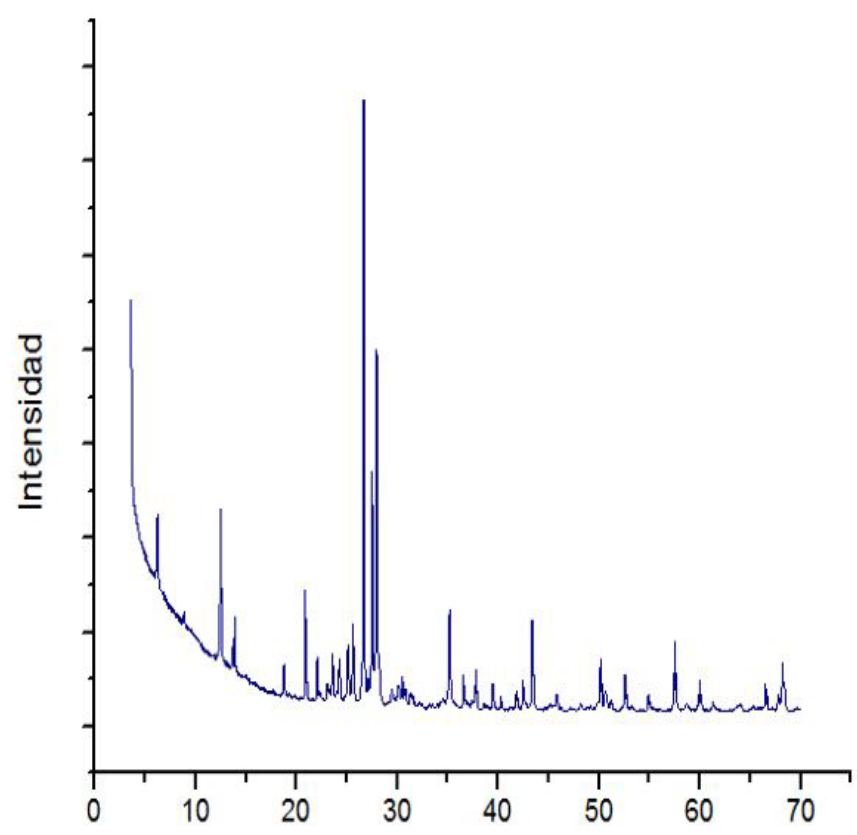

Figura 2. Difracción de rayos X de la muestra de arcilla proveniente de la mina La Alejandra del municipio de El Zulia 


\begin{tabular}{|c|c|c|}
\hline FÓRMULA & $\begin{array}{c}\text { FASE MINERALÓGICA } \\
\text { IDENTIFICADA }\end{array}$ & $\begin{array}{c}\text { CUANTIFICACIÓN } \\
\text { (\%) }\end{array}$ \\
\hline $\mathrm{SiO}_{2}$ & Cuarzo & 22,1 \\
\hline $\mathrm{KAl}_{2}\left(\mathrm{AlSi}_{3} \mathrm{O}_{10}\right)(\mathrm{OH})_{2}$ & Moscovita & 7,2 \\
\hline$\left(\mathrm{Mg}, \mathrm{Fe}^{+2}\right)_{5} \mathrm{Al}\left((\mathrm{OH})_{8} / \mathrm{AlSi}_{3} \mathrm{O}_{10}\right)$ & Clinocloro & 10,8 \\
\hline $\mathrm{Ca}\left(\mathrm{CO}_{3}\right)$ & Calcita & 1,7 \\
\hline $\mathrm{Na}\left(\mathrm{AlSi}_{3} \mathrm{O}_{8}\right)$ & Albita & 25,1 \\
\hline $\mathrm{Al}_{2}\left(\mathrm{Si}_{2} \mathrm{O}_{5}\right)(\mathrm{OH})_{4}$ & Caolinita & $<<1,0$ \\
\hline $\mathrm{K}\left(\mathrm{AlSi}_{3} \mathrm{O}_{8}\right)$ & Microclina & 13,4 \\
\hline $\mathrm{Na}_{-} \mathrm{Ca}_{-} \mathrm{Al}_{-}-\mathrm{Si}_{4} \mathrm{O}_{10}-\mathrm{O}$ & Montmorillonita & 10,0 \\
\hline $\mathrm{Mg}_{3}\left(\mathrm{Si}_{4} \mathrm{O}_{10}\right)(\mathrm{OH})_{2}$ & Vermiculita & 0,3 \\
\hline \multicolumn{2}{|c|}{ Total cristalino } & $\mathbf{9 0 , 6}$ \\
\hline \multicolumn{2}{|c|}{ Total amorfo } \\
\hline
\end{tabular}

Tabla I. Análisis mineralógico cuantitativo de la muestra de arcilla de la mina La Alejandra del municipio de El Zulia

De acuerdo con esto, las fases identificadas predominantes en la muestra son la albita y el cuarzo; además se observa la presencia en porcentajes considerables de microclina, clinocloro, montmorillonita y moscovita. La calcita se encuentra en un bajo porcentaje mientras que la caolinita y la vermiculita se encuentran en porcentajes por debajo del $1 \%$. Estos resultados no concuerdan con estudios previos realizados a materiales arcillosos de la formación Guayabo de la región, ya que en estos se presenta mayor contenido de cuarzo y de caolinita (Gelves, Monroy, Sánchez, \& Ramirez, 2013) (Mora Basto, 2015) (Cáceres, 2018). Se debe tener en cuenta que la muestra de arcilla fue obtenida de un límite, lo que puede explicar las diferencias en cuanto a su composición.

Al realizar el análisis mineralógico es realmente elevado el porcentaje de feldespatos presentes; tectosilicatos en los que parte de los átomos de silicio están sustituidos por aluminio, ya que en la arcilla se encuentran de $\mathrm{Na}$ (albita) y de K (microclina) que se engloban dentro de los feldespatos alcalinos (Barba et al., 2002).

La elevada cantidad de álcalis que presenta esta materia prima determina su facilidad para fundir y reaccionar con otros componentes y en la muestra analizada se observó que a temperaturas de cocción bajas se obtuvieron buenos resultados. Adicionalmente, los feldespatos alcalinos son los más empleados en la industria cerámica (Barba et al., 2002)

\section{Difracción de rayos $\mathrm{X}$ de la muestra de cenizas de car- bón}

Se realizó el análisis de difracción de rayos $\mathrm{X}$ de cada uno de los procesos de generación de cenizas (dosificación con pala y con carbojet) mostrando en la figura 3 los perfiles obtenidos.

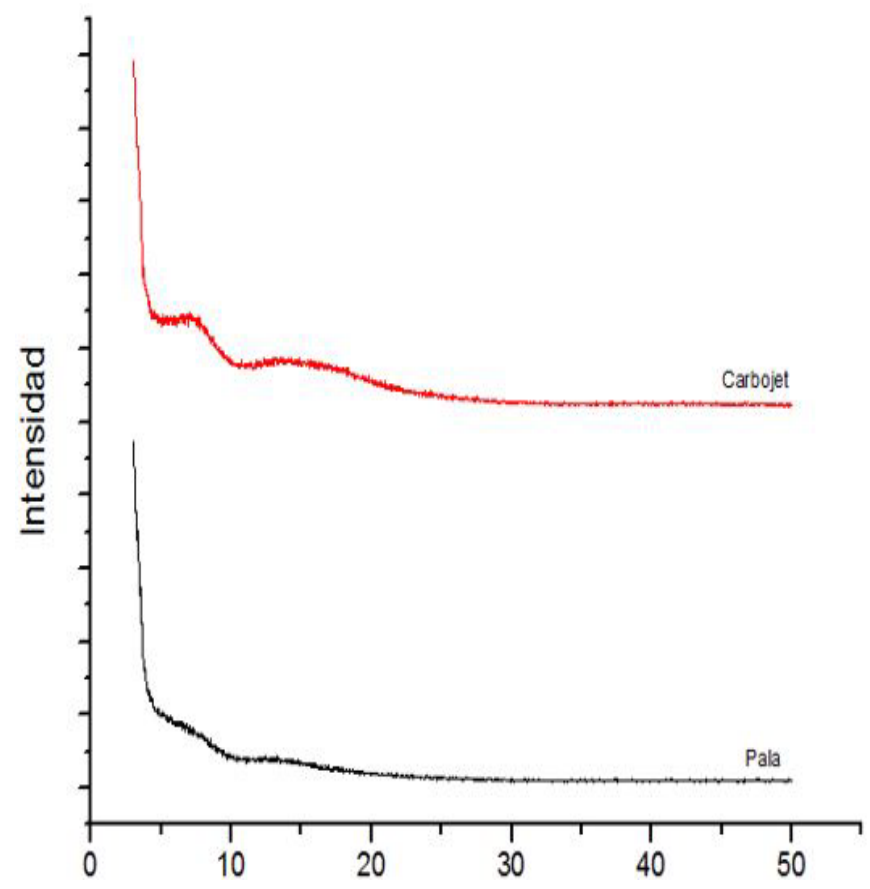

Figura 3. Difracción de rayos $\mathrm{X}$ de las muestras de cenizas de carbón 
El difractograma de las cenizas de carbojet muestra un pico centrado en $6,98^{\circ} 2 \theta$ con una distancia interplanar de 12,60 Å en el plano de reflexión 001 y otra señal menos intensa en $14,26^{\circ} 2 \theta$ con una distancia interplanar de $6,21 \AA \AA$ en el plano de reflexión 002. Mientras que para el perfil de las cenizas de pala se presenta un pico en $6,96^{\circ}$ $2 \theta$ con una distancia interplanar de $12,69 \AA$ y otro en $12,83^{\circ} 2 \theta$ con una distancia interplanar de $6,80 \AA$, en los planos de reflexión hkl (001) y (002), respectivamente.

De acuerdo con lo anterior, se puede decir que los perfiles de difracción obtenidos son característicos para carbón amorfo como lo son las cenizas, cuyos planos representativos son el hkl (001) y el hkl (002) lo que indica una estructura tipo grafito, debido a su empaquetamiento en el eje z (Peña \& Ortega, 2014) (Moreno Xavier; Ayora, Carles; Pereira, Constantino Fernández; Janssen-Jurkovicová, Maria, 2001)

\section{Espectroscopia infrarroja de la arcilla}

En la figura 4 se muestra el espectro FTIR-ATR normalizado obtenido para la muestra de arcilla utilizada.

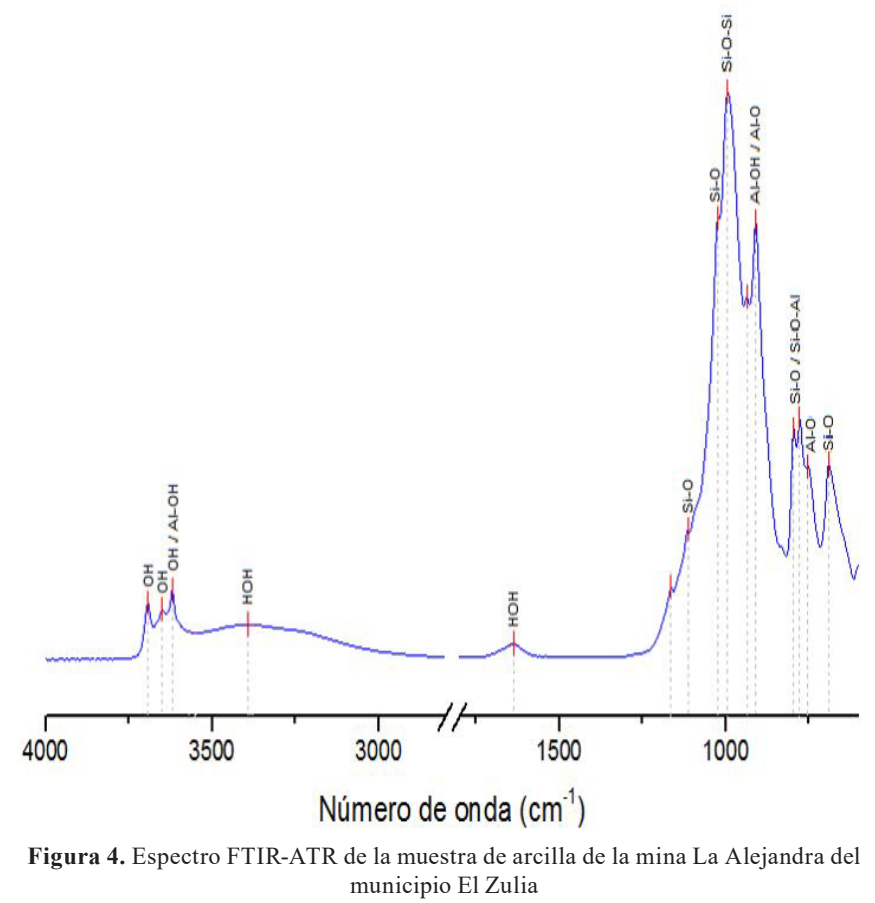

Los minerales de las arcillas se pueden diferenciar por la disposición de apilamiento de sus hojas y por la ocupación de los iones en los sitios octaédricos y tetraédricos. Por esto, para identificar un mineral de arcilla respecto a otro, se utiliza el análisis por FTIR, en el cual las bandas de absorción que producen los grupos $\mathrm{OH}$ estructurales y Si-O son fundamentales.

Se pueden observar bandas en el espectro infrarrojo en 3393 y $1647 \mathrm{~cm}-1$ referentes al estiramiento H-O-H del agua absorbida en la muestra (Madejová, 2003). La moscovita presenta una vibración de estiramiento de los grupos $\mathrm{OH}$ en $3618 \mathrm{~cm}-1$, así mismo la banda a $795 \mathrm{~cm}-1 \mathrm{se}$ debe a la vibración de flexión del enlace O-Si-O que confirma la presencia de moscovita (Nayak \& Singh, 2007).

En el espectro FTIR-ATR se pueden observar otras absorciones a $908 \mathrm{~cm}-1$ que corresponde a las vibraciones de flexión del enlace Al-Al-OH y otro pico en $1023 \mathrm{~cm}-1$ que hace referencia al estiramiento del enlace Si-O (Madejová, 2003).

La muestra de arcilla analizada presenta bandas de estiramiento y flexión del enlace Si-O en el intervalo de 1163 a $631 \mathrm{~cm}-1$, de las cuales la tensión asimétrica del enlace Si-O a 1163 y las vibraciones de estiramiento del enlace Si-O a 795 y $691 \mathrm{~cm}-1$, muestran la presencia de cuarzo. Los diferentes arreglos dentro de las capas de los minerales se reflejan en la forma y posición de las bandas (Madejová, 2003).

\section{Espectroscopia infrarroja de la ceniza}

Los espectros FTIR de las muestras de cenizas de carbón obtenidas ya sea por dosificación con pala o carbojet, muestran un comportamiento semejante, con bandas características de los minerales que la componen en 1083 cm-1 correspondiente a la vibración de estiramiento de Si-O del cuarzo, y un pico en $791 \mathrm{~cm}-1$ correspondiente al enlace $\mathrm{Al} / \mathrm{Si}-\mathrm{O}$ que puede hacer referencia a la presencia de mullita en las cenizas (Muñoz Lucas, 2012) (Kant, Saxena, Sarkar, Varma, \& Mishra, 2012). 


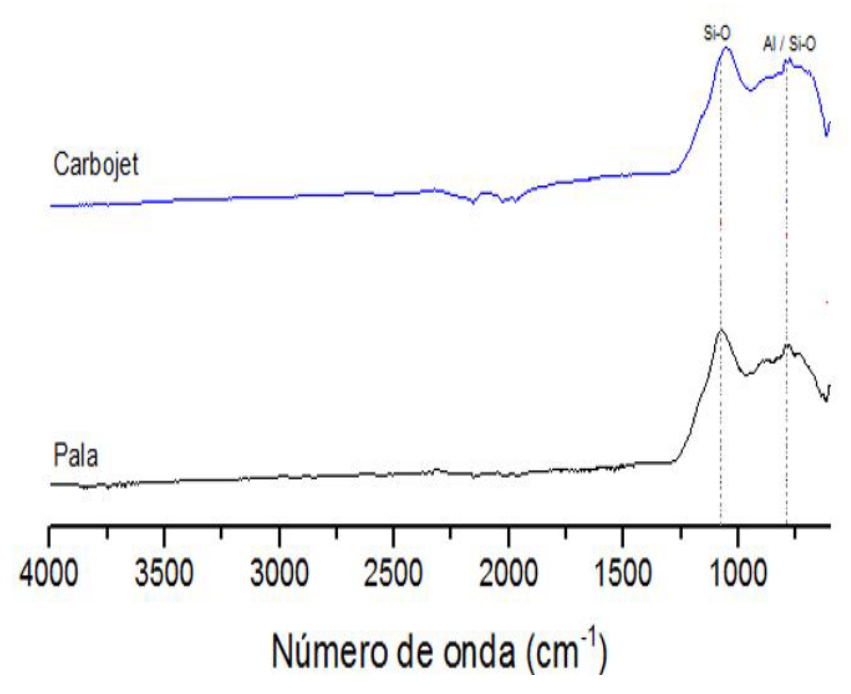

Figura 5. Espectro FTIR-ATR de las muestras de cenizas de carbón

\section{Comportamiento tecnológico}

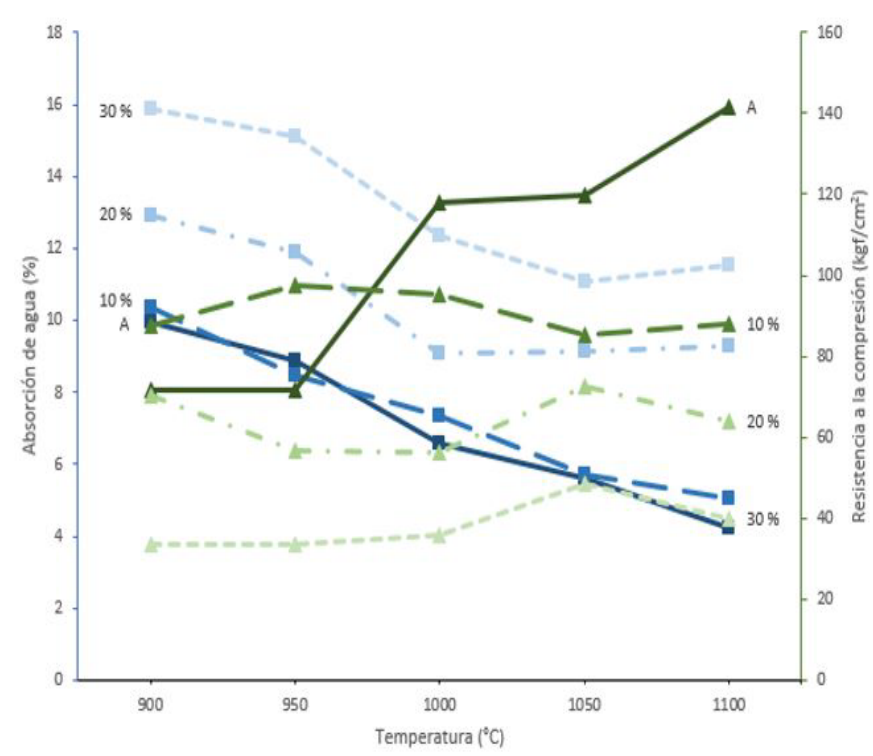

Figura 6. Absorción de agua y resistencia a la compresión de las mezclas elaboradas

La absorción de agua de una arcilla está determinada principalmente por el tipo y la proporción de los minerales que la componen. El análisis del comportamiento tecnológico muestra que se pueden elaborar bloques sinterizados a una temperatura de 900 y $950{ }^{\circ} \mathrm{C}$, mezclando arcilla y $10 \%$ de ceniza obteniendo resultados favorables en términos de aumento de la resistencia a la compresión con una ligera variación en el porcentaje de absorción de agua (figura 6). Esto demuestra que se reduce la temperatura tradicional de cocción de los bloques de cerámica en la región.
Al comparar los valores de absorción de agua y de resistencia a la compresión obtenidos en este estudio, con los establecidos en la norma NTC 4205 (ICONTEC, n.d.-b) para bloques no estructurales para interiores de perforación horizontal, se evidencia que estos cumplen dichos parámetros que permite porcentajes de absorción máximos de $17 \%$ en promedio y los resultados obtenidos tanto para los bloques de arcilla pura como para los de la mezcla son inferiores. Además, se logró cumplir con el parámetro de resistencia a la compresión ya que la norma establece un valor mínimo de $30 \mathrm{kgf} / \mathrm{cm} 2$ en promedio por muestra analizada con un valor mínimo de $20 \mathrm{kgf} /$ $\mathrm{cm} 2$ para cada unidad.

\section{Conclusiones}

Con la presente investigación se logró estudiar el efecto de la incorporación de un nutriente tecnológico como las cenizas de carbón provenientes de la combustión de los hornos colmena en una pasta cerámica, para evaluar su potencial uso y de esta manera contribuir con una alternativa para la utilización de este desecho de la industria cerámica.

Los resultados obtenidos permiten corroborar que el comportamiento tecnológico de la arcilla está estrechamente relacionado con la composición mineralógica y granulométrica, ya que los mejores resultados obtenidos se presentan a bajas temperaturas lo cual se debe a la cantidad de fundentes alcalinos presentes en la muestra de arcilla utilizada.

Los resultados muestran que el material fabricado presenta un porcentaje de absorción de agua y una resistencia a la compresión de acuerdo con los rangos establecidos por los estándares colombianos. Además, también es más liviano de lo habitual, lo que reduce los costos de transporte y se puede utilizar en los procesos de producción de materiales tradicionales de la región con un sello ecológico.

\section{Referencias}

[1] Arsenovic, M., Radojević, Z., Jakšić, Ž., \& Pezo, L. (2015). Mathematical approach to application of industrial wastes in clay brick production - Part II: Optimization. Ceramics International, 41(3), 48994905. https://doi.org/10.1016/j.ceramint.2014.12.050 
[2] Balaguera, L., \& Carvajal, J. (2004). Estudio para producir bloque aligerado a partir de mezclas de arcilla, cenizas volantes y poliestireno expandido en la empresa Cerámicas Támesis S.A. 1-81.

[3] Barba, A., Beltrán, V., Felíu, C., García, J., Ginés, F., Sánchez, E., \& Vicente., S. (2002). Materias primas para la fabricación de soportes de baldosas cerámicas (Segunda ed). Castellón: Instituto de Tecnología Cerámica.

[4] Barrera Castro, M. Y. (2009). Estudio físicomecánico de un material compuesto pigmentado de Pead/arcilla orgánicamente modificada. Universidad Industrial de Santander.

[5] Cáceres, V. I. (2018). Caracterización de material arcilloso proveniente de tres formaciones geológicas del área metropolitana de Cúcuta y su potencial aplicación en cerámica. Universidad de Pamplona.

[6] Castells, X. E. (2015). Nutrientes tecnológicos para la industria cerámica. UNIVERSIDAD DE JAÉN.

[7] Çiçek, T., \& Çinçin, Y. (2015). Use of fly ash in production of light-weight building bricks. Construction and Building Materials, 94, 521-527. https://doi.org/10.1016/j. conbuildmat.2015.07.029

[8] Consoli, N. C., Rocha, C. G. Da, \& Saldanha, R. B. (2014). Coal fly ash-carbide lime bricks: An environment friendly building product. Construction and Building Materials, 69, 301-309. https://doi.org/10.1016/j. conbuildmat.2014.07.067
[9] Díaz Fuentes, C. X. (2014). Elaboración y caracterización de un material compuesto, de matríz polvos de arcilla atomizados y refuerzo residuos de la combustión del carbón, conformado por prensado uniaxial. Universidad Francisco de Paula Santander: Cúcuta.

[10] Durán Angarita, E. J., \& Pinto Gelvez, Y. X. (2001). Estudio de factibilidad técnicoeconómico de utilización de residuos de lodos de cal y tierras diatomáceas de la cervecería Bavaria de Cúcuta, mezclados con arcilla para la fabricación de bloques en cerámicas Támesis S.A in Ingeniería de producción indust. Universidad Francisco de Paula Santander: Cúcuta.

[11] Gelves, J. F., Monroy, R., Sánchez, J., \& Ramirez, R. P. (2013). Estudio comparativo de las técnicas de extrusión y prensado como procesos de conformado de productos cerámicos de construcción en el Área Metropolitana de Cúcuta. Boletin de La Sociedad Espanola de Ceramica y Vidrio, 52(1), 48-54. https://doi. org/10.3989/cyv.62013

[12] ICONTEC. (n.d.-a). Norma técnica colombiana NTC-4017. Ingeniería Civil y Arquitectura. Métodos para muestreo. Ensayos de unidades de mampostería de arcilla. Bogotá.

[13] ICONTEC. (n.d.-b). Norma técnica colombiana NTC 4205. Ingeniería civil y arquitectura. Unidades de mampostería de arcilla cocida. Ladrillos y bloques cerámicos. Bogotá.

[14] Kant, U., Saxena, V. K., Sarkar, A., Varma, A., \& Mishra, K. (2012). Characterization of Coal ash from a Captive Power plant for Potential End uses. Thessaloniki Greece, 10(9), 25-27.

[15] Kockal, N. (2012). Utilization of different types of coal fly ash in the production of ceramic tiles. Boletín de La Sociedad Española de Cerámica y Vidrio, 297-304. 
[16] Lafarge. (2014). Hoja de datos de seguridad de materiales. HDSM: Cenizas volantes y cenizas de fondo Lafarge.

[17] Madejová, J. (2003). Review: FTIR techniques in clay mineral studies. Vibrational Spectroscopy, $31,1-10$.

[18] Monroy, R., \& Mora, S. (2005). Estudio para producir termoarcilla con mezclas de arcilla, poliestireno y cenizas. Universidad Francisco de Paula Santander: Cúcuta.

[19] Mora Basto, R. L. (2015). Caracterización de arcillas provenientes de la mina Murano del municipio El Zulia, Norte de Santander, Colombia. Universidad de Pamplon.

[20] Moreno Xavier; Ayora, Carles; Pereira, Constantino Fernández; Janssen-Jurkovicová, Maria, N. Q. (2001). 2. Caracterización De Las Cenizas Volantes. Utilization of Zeolites Synthesized from Coal Fly Ash for the Purification of Acid Mine Waters, 33-72.

[21] Muñoz Lucas, M. I. (2012). Caracterización y acondicionamiento de cenizas volantes para la eliminación de metales pesados en aguas contaminadas. 324.

[22] Nayak, P. S., \& Singh, B. K. (2007). Instrumental characterization of clay by XRF, XRD and FTIR. Bolletin of Materials Science, 30(3), 235-238.

[23] Peña, G., \& Ortega, L. (2014). Caracterización morfológica y estructural de polvo de cenizas volantes. 14(2), 14-19.

[24] Rozo, S. (2013). Evaluación de los cambios en las propiedades térmicas y mecánicas del bloque \# 5 fabricado en la región, según las mezclas entre arcilla y residuos industriales. Universidad Francisco de Paula Santander: Cúcuta.
[25] Sánchez, J., \& Díaz, J. I. (2011). Introducción a los hornos utilizados en la industria cerámica tradicional (Universida). Colombia.

[26] Sánchez Molina, J., Corpas, F. A., \& Álvarez Rozo, D. C. (2019). Aplicaciones de los nutrientes tecnológicos en la industria cerámica del área metropolitana de Cúcuta (Ecoe Edici). Bogotá: Universidad Francisco de Paula Santander.

[27] Sánchez Molina, J., \& Ramírez Delgado, P. (2013). El cluster de la cerámica del Área Metropolitana de Cúcuta (Primera). Cúcuta Norte de Santander: Universidad Francisco de Paula Santander.

[28] Sarabia, A., Ramirez, R., \& Sánchez, J. (2018). Use of central thermoelectric plant's coal fly ash for the production of ceramic tiles in the metropolitan area of Cúcuta. IEEE Explore, 1-7.

[29] Shoval, S., \& Beck, P. (2005). THERMO-FTIR SPECTROSCOPY ANALYSIS AS A METHOD OF CHARACTERIZING ANCIENT CERAMIC TECHNOLOGY. Journal of Thermal Analysis and Calorimetry, 1-8.

[30] Vidal, D., Torres, J., Mejia, R., \& Gonzalez, L. (2012). Estudio comparativo de cenizas de bagazo de caña como adicion puzolanica. Revista Colombiana de Materiales, 93-99.

[31] Yang, L., Shili, Z., Shuhua, M., Chunli, L., \& Xiaohui, W. (2017). Ceramic tiles derived from coal fly ash: Preparation and mechanical characterization. Ceramics International, 1195311966. 